\title{
Asymptotic time behaviour for non-autonomous degenerate parabolic problems with forcing term.
}

\author{
F. Ragnedda ${ }^{1}$, S. Vernier Piro ${ }^{2}$ and V. Vespri ${ }^{3}$
}

\begin{abstract}
We consider a non-autonomous, degenerate parabolic problem with Dirichlet boundary condition. We study the asymptotic behaviour of solutions, extending an earlier result of the authors, where the forcing term was taken zero.
\end{abstract}

AMS (MOS) subject classification: 35K55, 35B40, 35K65.

Keywords: Degenerate parabolic equation, asymptotic behaviour.

\section{Introduction}

Let $u(x, t)$ be the weak solution of the following initial boundary value problem

$$
\begin{gathered}
u_{t}=\operatorname{div} \mathbf{A}(x, t, u, \nabla u)+B(x, t, u, \nabla u), \quad(x, t) \in \Omega \times(t>0), \\
u(x, t)=0, \quad(x, t) \in \partial \Omega \times(t>0), \\
u(x, 0)=u_{0}(x) \geq 0, \quad x \in \Omega,
\end{gathered}
$$

where $\Omega$ is a bounded domain in $\mathbb{R}^{N}$ with $C^{1}$ boundary, $u_{0} \in L^{2}(\Omega)$ and $\int_{\Omega} u_{0}(x) d x>0 . B$ is called the forcing term. The functions $\mathbf{A}:=\left(A_{1}, \ldots, A_{N}\right)$

\footnotetext{
${ }^{1}$ Dipartimento di Matematica e Informatica, Viale Merello 92, 09123 Cagliari (Italy), ragnedda@unica.it

${ }^{2}$ Dipartimento di Matematica e Informatica, Viale Merello 92, 09123 Cagliari (Italy), svernier@unica.it

${ }^{3}$ Dipartimento di Matematica "U. Dini", viale Morgagni 67/a, 50134 Firenze (Italy) vespri@math.unifi.it)
} 
and $B(x, t, u, \nabla u)$ are assumed to be only measurable and to satisfy the following structure conditions:

$$
\begin{gathered}
\mathbf{A}(x, t, u, \nabla u) \nabla u-B(x, t, u, \nabla u) u \geq c_{0}|\nabla u|^{p}, \\
|\mathbf{A}(x, t, u, \nabla u)| \leq c_{1}|\nabla u|^{p-1},
\end{gathered}
$$

and

$$
|B(x, t, u, \nabla u)| \leq c_{2}|\nabla u|^{p-1}
$$

with $p>2$ and $c_{0}, c_{1}, c_{2}$ given positive constants.

A function $u \in C_{l o c}\left(\mathbb{R}^{+} ; L_{l o c}^{2}(\Omega)\right) \cap L_{l o c}^{p}\left(\mathbb{R}^{+} ; W_{l o c}^{1, p}(\Omega)\right)$ is a weak solution of (1.1)-(1.3), if $\forall \tau>0$

$$
\begin{gathered}
\int_{0}^{\tau} \int_{\Omega} u_{t} \phi d x d t+\int_{0}^{\tau} \int_{\Omega} \mathbf{A}(x, t, u, \nabla u) \nabla \phi d x d t \\
+\int_{0}^{\tau} \int_{\Omega} B(x, t, u, \nabla u) \phi d x d t=0
\end{gathered}
$$

for all bounded testing functions $\phi \in W_{l o c}^{1,2}\left(\mathbb{R}^{+} ; L^{2}(\Omega)\right) \cap L_{l o c}^{p}\left(\mathbb{R}^{+} ; W_{0}^{1, p}(\Omega)\right)$.

In the last few years, several papers were devoted to the study of the asymptotic behaviour of solutions to the porous media and the $p$-Laplace equations. We refer the reader to the recent monography by Vazquez ([11]) and to the references therein. To our knowledge, in all these references the Authors use elliptic results to study the asymptotic behaviour of the solutions. If, from one side, this makes the proof simple and very elegant, on the other hand it looks like this method cannot be applied in the case of time-dependent coefficients. In a recent paper ([8]) the Authors followed an alternative approach introduced by Berryman-Holland ([1]) and used in the context of the asymptotic behaviour of solutions to degenerate parabolic equations in [7] and [9]. This approach is more parabolic of the previous one, namely, relying on the properties of the evolution equations, it is possible to study the asymptotic behaviour of the solutions and derive the elliptic properties of the asymptotic limit as a by-product. Using this alternative approach and under the assumption $B=0$, in [8] the Authors studied the asymptotic behaviour of the solutions of a degenerate parabolic equations with time dependent coefficients. In this note we are able to remove the condition $B \neq 0$ even if we are compelled to assume the condition $u_{0} \in L^{2}(\Omega)$. We refer to [8] for more details and references. 


\section{Notations and preliminary results}

We recall some Lemmata we will need to prove the main results.

Let $\mathcal{A}$ be a domain of $\mathbb{R}^{N}$ and let $|\mathcal{A}|$ denote the Lebesgue measure of the set $\mathcal{A}$. If $f$ is a function defined in $\Omega$ with values in $\mathbb{R}$ and $c \in \mathbb{R}$, with $|f(x)>c|$, we denote the measure of the set $\mathcal{A}=\{x \in \Omega$ such that $f(x)>c\}$. For $\rho>0$, let $B_{\rho}(x) \subset \Omega$ be the ball centered at $x$ of radius $\rho$, and $B_{\rho}=B_{\rho}(0)$.

Lemma 2.1 (A Measure Theory Lemma, [4]) Let $u_{0} \in W^{1,1}\left(B_{\rho}\right)$ satisfy

$$
\left\|u_{0}(x)\right\|_{W^{1,1}\left(B_{\rho}\right)} \leq \gamma \rho^{N-1}, \quad\left|u_{0}(x)>\beta\right| \geq \alpha\left|B_{\rho}\right|,
$$

for some $\gamma \geq 0, \beta \in R$ and $\alpha \in(0,1)$. Then $\forall \delta \in(0,1)$ and $\lambda<\beta$, $\exists x_{0} \in B_{\rho}$ and $\eta=\eta(\alpha, \beta, \gamma, \delta, \lambda, N)$ such that

$$
\left|B_{\rho \eta}\left(x_{0}\right) \cap\left\{u_{0} \geq \lambda \beta\right\}\right| \geq(1-\delta)\left|B_{\rho \eta}\left(x_{0}\right)\right| .
$$

We consider now a solution $u(x, t)$ of (1.1)-(1.3). We introduce the set

$$
Q_{\rho, \tau}\left(x_{0}, t_{0}\right):=B_{\rho}\left(x_{0}\right) \times\left(t_{0}, t_{0}+\tau\right),
$$

with $Q_{\rho, \tau} \subset \Omega \times(t>0)$ and a piecewise smooth cutoff function $\zeta, 0 \leq \zeta \leq 1$, such that $|\nabla \zeta|<+\infty$ and $\zeta(x, t)=0$ if $x \notin B_{\rho}\left(x_{0}\right)$. We recall now the energy estimate with $\left(x_{0}, t_{0}\right)=(0,0)$.

For the proof of the following result we refer the reader to the monograph [2] pag.24.

Lemma 2.2 (Local energy estimates) Let $u$ be a local weak solution of (1.1)-(1.3) under the structure conditions (1.4)-(1.5). For all $t \in(0, \tau)$, $\exists C=C(p)>0$ such that for all cylinders $Q_{\rho, \tau}(0,0) \subset \Omega \times(0, \tau)$ and $\forall k \in \mathbb{R}$

$$
\begin{gathered}
\sup _{(0, \tau)} \int_{B_{\rho} \times\{t\}}(u-k)_{-}^{2} \zeta^{p} d x+\int_{0}^{\tau} \int_{B_{\rho}}\left|\nabla(u-k)_{-}\right|^{p} \zeta^{p} d x d t \\
\leq \int_{B_{\rho} \times\{0\}}(u-k)_{-}^{2} \zeta^{p} d x+C \int_{0}^{\tau} \int_{B_{\rho}}(u-k)_{-}^{p}|\nabla \zeta|^{p} d x d t+ \\
+p \int_{0}^{\tau} \int_{B_{\rho}}(u-k)_{-}^{2} \zeta^{p-1} \zeta_{t} d x d t .
\end{gathered}
$$


Moreover we will use a variant of a DeGiorgi-like Lemma (see [5]). We have assumed that $u_{0}$ is non-negative. For a fixed cylinder

$$
Q_{2 \rho, \theta(2 \rho)^{p}}\left(x_{0}, t_{0}\right):=B_{2 \rho}\left(x_{0}\right) \times\left(t_{0}, t_{0}+\theta(2 \rho)^{p}\right) \subset \Omega \times(t>0),
$$

with $\theta>0$, let $\mu_{ \pm}$and $\omega$ be two non negative numbers such that

$$
\mu_{+} \geq \underset{Q_{2 \rho, \theta(2 \rho)^{p}}}{\operatorname{ess} \sup } u, \quad \mu_{-} \leq \underset{Q_{2 \rho, \theta(2 \rho)^{p}}}{\operatorname{essinf}} u, \quad \omega \geq \mu_{+}-\mu_{-} .
$$

Denote by $\lambda$ and $a$ fixed numbers in $(0,1)$ and by $Q_{\rho}(\theta)=B_{\rho} \times\left(0, \theta \rho^{p}\right)$.

Lemma 2.3 ([5], see also [2] pag. 49) Let $u$ be a local weak solution of (1.1)-(1.3) under the structure conditions (1.4)-(1.5) and let $0<a<1$. There exists a number $\nu>0$ depending upon $\theta, \lambda, \omega$, and the data such that if

$$
\left|\left(u \leq \mu_{-}+\lambda \omega\right) \cap Q_{2 \rho}(\theta)\right| \leq \nu\left|Q_{2 \rho}(\theta)\right|,
$$

then

$$
u \geq \mu_{-}+a \lambda \omega, \text { a.e. in } Q_{\rho}(\theta) \text {. }
$$

We remark that in [5] the result of Lemma 2.3 is stated in a more general form; here we simplify it, according to our hypotheses.

Under the same conditions of the previous Lemmata, we have this variant of Lemma 2.3

Lemma 2.4 ([5]) Let $u$ be a local weak solution of (1.1)-(1.3) under the structure conditions (1.4)-(1.5) and let $\lambda$ and $\beta$ be two positive numbers, with $0<\lambda<1$ such that

$$
u\left(x, t_{0}\right) \geq \lambda \beta, \quad \text { a.e. } x \in B_{2 \rho}
$$

then $\forall a \in(0,1)$

$$
u(x, t) \geq a \lambda \beta, \quad \text { a.e. in } B_{\rho} \times\left(t_{0}, t_{0}+\theta(2 \rho)^{p}\right),
$$

with $\theta=\frac{\delta}{(\lambda \beta)^{p-2}}$, and $\delta \in(0,1)$ is a quantity that depends only on a, and the data. 
Lemma 2.5 ([5], sec.5) Let $u$ be a local weak solution of (1.1)-(1.3) under the structure conditions (1.4)-(1.5) and let $\lambda$ and $\beta$ be two positive numbers, with $0<\lambda<1$ such that

$$
u(x, 0) \geq \lambda \beta, \quad \text { a.e. } x \in B_{2 \rho} ;
$$

then $\forall t_{0}>0$ and $\forall t \geq t_{0}>0$

$$
u(x, t) \geq \gamma_{0} \frac{\lambda \beta}{(t)^{\frac{1}{p-2}}}, \text { a.e. } x \in B_{\rho},
$$

where $\gamma_{0}$ depends upon the data and $t_{0}$.

\section{Estimate from above and below.}

We show in this section that the solution of (1.1)-(1.3) is bounded both from above and from below under structure conditions (1.4)-(1.6).

\section{Estimate from above.}

Theorem 3.1 Let $u$ be the solution of (1.1)-(1.6). Let $t_{0}>0$. Then there exists a constant $C_{1}>0$ depending only upon the data and $t_{0}$, such that for any $t>t_{0}$, and for any $x \in \Omega$

$$
u(x, t) \leq C_{1} t^{-\frac{1}{p-2}}
$$

Proof - Following the same pattern of [3], [6] and [7], we choose in (1.7), $\phi=u$. Integrating in the space variables and applying the structure condition (1.4), it follows that

$$
\frac{1}{2} \frac{d}{d t}\|u\|_{L^{2}}^{2}=\int_{\Omega}(-\mathbf{A}(x, t, u, \nabla u) \nabla u+B u) d x \leq-c_{0} \int_{\Omega}|\nabla u|^{p} d x .
$$

Moreover we estimate the $L^{p}$-norm of the gradient by using Hölder and Sobolev inequalities to get

$$
\int_{\Omega}|\nabla u|^{p} d x \geq C\left(\int_{\Omega} u^{2} d x\right)^{p / 2},
$$

$C$ a positive constant depending on $|\Omega|, p$ and $N$. 
By (3.2) and (3.3), we have

$$
\frac{1}{2} \frac{d}{d t}\|u\|_{L^{2}}^{2} \leq-C\left(\int_{\Omega} u^{2} d x\right)^{p / 2} .
$$

For any $t>0$, the function $\psi:=\int_{\Omega} u^{2} d x$ satisfies the ordinary differential inequality

$$
\dot{\psi}+2 C \psi^{p / 2} \leq 0
$$

By integrating (3.5) we get that

$$
\|u(t)\|_{L^{2}} \leq C_{2} t^{-\frac{1}{p-2}} .
$$

Therefore by Theorem 4.3, pag 123 of [2] one gets (3.1) with the constant $C_{1}$ depending upon the $L^{2}$ norm of the initial datum (see [8] for more details). We remark that in [8] the initial datum was assumed to belong only to $L^{1}$ and in order to obtain the validity of Theorem 3.1 $B$ in (1.1) was zero.

\section{Estimate from below.}

First we note that in [5], [6] and [7], in order to prove estimate from below, the authors use inequalities coming from the Rayleigh quotient. Here we assume the coefficients $\mathbf{A}$ and $B$ to be time-dependent and therefore we derive a new method mainly based on energy estimates (2.2).

Theorem 3.2 Let $u$ be the solution of (1.1)-(1.6). There exist $t_{1}>0$, two constant $C_{3}>0$ and $r>0$, and a point $x_{0} \in \Omega$ depending only upon the data and upon $t_{1}$, such that for any $t>t_{1}$, and for any $x \in B\left(x_{0}, r\right) \subset \Omega$

$$
u(x, t) \geq C_{3} t^{-\frac{1}{p-2}}
$$

Proof - Since $\int_{\Omega} u_{0}>0$, we know that there exist $\nu, \lambda, R$ and $x_{0}$ such that

$$
\left|B_{R}\left(x_{0}\right) \cap[u \geq \lambda]\right| \geq \nu\left|B_{R}\left(x_{0}\right)\right|, \quad B_{R}\left(x_{0}\right) \subset \Omega .
$$

Assume $x_{0}=0$. Applying energy estimates in Lemma 2.2 in the time interval $(0, \tilde{t})$ and following [8], we obtain

$$
\left|B_{(1-\epsilon) R} \cap[u \geq \lambda \beta]\right| \geq \nu\left|B_{(1-\epsilon) R}\right|, \quad \forall t \in(0, \tilde{t}),
$$


with $\beta$ a suitable positive constant.

Now we fix an interval $\left[\frac{\tilde{t}}{2}, \tilde{t}\right]$ and in order to apply Lemma 2.1, for a time $t \in\left[\frac{\tilde{t}}{2}, \tilde{t}\right]$, we prove that $u \in W_{0}^{1, p}(\Omega)$.

We have already pointed out that for each $t, u(t) \in L^{2}(\Omega)$, since $\Omega$ is bounded. To prove that there exists a $\tilde{t} / 2<t<\tilde{t}$, such that $\nabla u(t) \in L^{p}(\Omega)$, we start from (1.7) with $\phi=u$, to derive

$$
\begin{gathered}
\int_{\tilde{t} / 2}^{\tilde{t}} \int_{\Omega} u u_{t} d x d t=\int_{\tilde{t} / 2}^{\tilde{t}} \int_{\Omega} u(\operatorname{div} \mathbf{A}+B) d x d t \\
=\int_{\tilde{t} / 2}^{\tilde{t}} \int_{\Omega}(-\mathbf{A} \nabla u+B u) d x d t \leq-c_{0} \int_{\tilde{t} / 2}^{\tilde{t}} \int_{\Omega}|\nabla u|^{p} d x d t
\end{gathered}
$$

where we have used the structure condition (1.4).

By integrating in time the left hand part of (1.7) and by (3.9), we deduce

$$
\frac{1}{2} \int_{\Omega} u^{2}(\tilde{t}) d x-\frac{1}{2} \int_{\Omega} u^{2}(\tilde{t} / 2) d x \leq-c_{0} \int_{\tilde{t} / 2}^{\tilde{t}} \int_{\Omega}|\nabla u|^{p} d x d t,
$$

which yields

$$
c_{0} \int_{\tilde{t} / 2}^{\tilde{t}} \int_{\Omega}|\nabla u|^{p} d x d t \leq \int_{\Omega} u^{2}(x, \tilde{t} / 2) d x \leq\left(\int_{\Omega} u_{0}^{2}(x) d x\right) .
$$

This means that there exists at least one level $\bar{t} \in\left[\frac{\tilde{t}}{2}, \tilde{t}\right]$ where $|\nabla u(\bar{t})| \in$ $L^{p}(\Omega)$. Then at $t=\bar{t}, u(\cdot, \bar{t}) \in W^{1, p}(\Omega)$ and satisfies (3.8): consequently $u$ satisfies the Lemma 2.1 and this implies that there exists a ball $B_{\rho^{\prime}} \subset$ $B_{(1-\epsilon) R}$, such that $\forall \delta^{\prime} \in(0,1)$

$$
\left|\left\{u \geq \frac{\lambda \beta}{2}\right\} \cap B_{\rho^{\prime}}\right| \geq\left(1-\delta^{\prime}\right)\left|B_{\rho^{\prime}}\right| .
$$

From (3.11) we have

$$
\left|\left\{u \leq \frac{\lambda \beta}{2}\right\} \cap B_{\rho^{\prime}}\right| \leq \delta^{\prime}\left|B_{\rho^{\prime}}\right| .
$$

Using again the energy estimates Lemma 2.2, we can expand (3.12) in time and reach a level $\bar{t}^{\prime} \geq \bar{t}$ such that for any $t \in\left[\bar{t}, \bar{t}^{\prime}\right]$ 


$$
\left|\left\{u \geq \frac{\lambda \beta \beta^{\prime}}{2}\right\} \cap B_{(1-\epsilon) \rho^{\prime}}\right| \geq\left(1-2 \delta^{\prime}\right)\left|B_{(1-\epsilon) \rho^{\prime}}\right| .
$$

and

$$
\left|\left\{u \leq \frac{\lambda \beta \beta^{\prime}}{2}\right\} \cap B_{(1-\epsilon) \rho^{\prime}}\right| \leq\left(2 \delta^{\prime}\right)\left|B_{(1-\epsilon) \rho^{\prime}}\right|
$$

Arguing as in [8] (to which we refer the reader for more details) we get (3.7), using the results in [2], pag 49 and in [3].

\section{Large time behaviour.}

In this section we investigate the behavior of the solution of (1.1)-(1.3) for large times. We work as in [5] and we set

$$
w(x, t)=u(x, t) t^{\frac{1}{p-2}}
$$

and with $t=e^{\tau}, w(x, \tau)$ is a solution of the equation

$$
w_{\tau}=\operatorname{div} \tilde{A}(x, \tau, w, \nabla w)+\tilde{B}(x, \tau, w, \nabla w)+\frac{1}{p-2} w
$$

with

$$
w(x, 0)=u_{0}(x)
$$

$\operatorname{In}(4.2)$

$$
\tilde{\mathbf{A}}=\mathbf{A} e^{\tau\left(\frac{p-1}{p-2}\right)}, \quad \tilde{B}=B e^{\tau\left(\frac{p-1}{p-2}\right)}
$$

Starting from estimates (3.1) and (3.7) and arguing as in [8] (to which we refer for more details), we are able to prove the following results:

Theorem 4.1 For any compact $K \subset \subset \Omega$, there exist a time $t_{1}$ and positive constants $C_{4}-C_{6}$, depending only upon the data, $t_{1}$ and the compact $K$ such that

- for each $x \in K$, and for each $t \geq t_{1}$

$$
C_{4} \leq w(x, t)=u(x, t) t^{\frac{1}{p-2}} \leq C_{5} ;
$$


- $w$ is uniformly $\alpha$-Hölder continuous with the Hölder continuity constant that depends only upon the data and

$$
\|w\|_{C^{\alpha, \frac{\alpha}{p}}\left(\Omega,\left[t_{1}, \infty\right]\right)} \leq C_{6}
$$

Denote with $d(x)$ the distance from the point $x$ to the boundary $\partial \Omega$.

Theorem 4.2 There exist two constants $\beta \in(0,1)$ and $\gamma_{9}>0$, such that for each positive number $d$ there exists a time $t_{d}$ such that for each $x \in \Omega$ with $d(x) \geq d$, and for each $t \geq t_{d}$ we have

$$
\gamma_{9} d(x)^{\beta} \leq w(x, t)=u(x, t) t^{\frac{1}{p-2}} .
$$

By Theorem 4.1, $w(t)$ is equiHölder continuous, therefore, up to a subsequence, there is a function $v \in C^{\alpha}(\Omega)$ such that $w \rightarrow v$ in $C^{\alpha}$.

If we want the function $v$ to be the solution of a suitable partial differential equation, we have to assume some hypotheses on the coefficients $\tilde{A}$ and $\tilde{B}$ in (4.3).

(4.8) $\exists$ a function $H(x, \tau, w, p)$ such that $\frac{\partial H}{\partial p_{i}}=\tilde{A}_{i}, \quad \frac{\partial H}{\partial w}=-\tilde{B}$,

$$
\begin{array}{r}
\exists \lim _{\tau \rightarrow+\infty} \tilde{\mathbf{A}}(x, \tau, w, p)=\mathbf{A}_{\infty}(x, w, p), \\
\exists \lim _{\tau \rightarrow+\infty} \tilde{B}(x, \tau, w, p)=B_{\infty}(x, w, p), \\
\text { such that } \frac{\partial H}{\partial p_{i}}=\tilde{A}_{i}, \quad \frac{\partial H}{\partial w}=-\tilde{B}, \\
\text { and } \frac{\partial H}{\partial \tau} \leq 0, \\
\int_{\Omega} H(x, \tau, w, \nabla w) d x \geq C_{1} .
\end{array}
$$

Theorem 4.3 Assume that hypotheses (3.5)-(3.9) hold. Then the function $v$ belongs to $W_{0}^{1, p} \cap L^{2}(\Omega)$ and it is a non trivial solution of

$$
\operatorname{div}\left(\mathbf{A}_{\infty}(x, v, \nabla v)\right)+B_{\infty}(x, v, \nabla v)=\frac{1}{p-2} v
$$

Proof - The functional

$$
F(x, \tau, w(x, \tau), \nabla w(x, \tau))=\int_{\Omega} H(x, \tau, w, \nabla w) d x-\frac{1}{2(p-2)} \int_{\Omega} w^{2}(x, \tau) d x
$$


is monotone decreasing in time. In fact

$$
\begin{gathered}
\frac{d}{d \tau} \int_{\Omega} H d x=\int_{\Omega}\left[\frac{\partial H}{\partial p_{i}} \nabla_{i} w_{\tau}+\frac{\partial H}{\partial w} w_{\tau}+\frac{\partial H}{\partial \tau}\right] d x \\
\leq \int_{\Omega} \frac{\partial H}{\partial p_{i}} \nabla_{i} w_{\tau} d x+\frac{\partial H}{\partial w} w_{\tau}=-\int_{\Omega}(\operatorname{div} \tilde{\mathbf{A}}+\tilde{B}) w_{\tau} d x \\
=-\int_{\Omega}\left(w_{\tau}\right)^{2} d x+\frac{1}{p-2} \int_{\Omega} w w_{\tau} d x .
\end{gathered}
$$

Then

$$
\frac{d F}{d \tau} \leq-\int_{\Omega}\left(w_{\tau}\right)^{2} \leq 0
$$

As the functional $F$ is bounded from below, this implies that, up to a subsequence, $F_{\tau}$ (and therefore $\int_{\Omega} w_{\tau}^{2}(x) d x$ ) converges to zero.

Then $w$ converges to its limit $v \in W_{0}^{1, p} \cap L^{2}(\Omega)$ and $v$ is the solution of (4.11).

\section{References}

[1] J.G. Berryman, C.J. Holland, Stability of the separable solution for fast diffusion, Arch. Rational Mech. Anal., 74, (1980), no. 4, 379-388.

[2] E. Di Benedetto, Degenerate parabolic equations, Springer Verlag, (1993)

[3] E. Di Benedetto, J.M.Urbano, V.Vespri, Current Issues on Singular and Degenerate Evolution Equations, Evolutionary equations. Vol.1, Handb. Differ. Equ., North-Holland, Amsterdam, (2004), 169-286.

[4] E. Di Benedetto, U. Gianazza, V. Vespri, Local Clustering of the Non Zero Set of Functions in $W^{1,1}(E)$, Rend. Accad. Naz. Lincei, Roma, 17 (2006), 223-225.

[5] E. Di Benedetto, U. Gianazza, V. Vespri, Harnack Estimates for QuasiLinear Degenerate Parabolic Differential Equations, Acta Math., 200, (2008), no. 2, 181-209.

[6] U. Gianazza, V. Vespri, A Harnack Inequality for a Degenerate Parabolic Equation, Journal of Evolution Equations, 97 (1998), 3-22. 
[7] J.J. Manfredi, V. Vespri, Large time behaviour of solutions to a class of Doubly Nonlinear Parabolic equations, Electronic J. Diff. Eq., (1994), 2, $1-1 \%$

[8] F. Ragnedda, S. Vernier Piro, V. Vespri, Large time behaviour of solutions to a class of nonautonomus degenerate parabolic equations, preprint Dip.Matematica e Inf., Universitá di Cagliari, (2008).

[9] G. Savaré, V. Vespri, The asymptotic profile of solutions of a class of doubly nonlinear equations, Nonlinear Anal., 22, (1994), no. 12, 15531565 .

[10] J.L. Vázquez, Asymptotic behaviour and propagation properties of the one-dimensional flow of gas in a porous medium, Trans. Amer. Math. Soc., 277, (1983), no. 2, 507-527.

[11] J.L. Vázquez, Smoothing and decay estimates for nonlinear diffusion equations. Equations of porous medium type, Oxford Lecture Series in Mathematics and its Applications, 33, Oxford University Press, Oxford, 2006. 